\title{
Higher emotional intelligence is related to lower test anxiety among students
}

\author{
This article was published in the following Dove Press journal: \\ Neuropsychiatric Disease and Treatment \\ 12 January 2016 \\ Number of times this article has been viewed
}

\author{
Mohammad Ahmadpanah' \\ Mohammadreza Keshavarz' \\ Mohammad Haghighi' \\ Leila Jahangard' \\ Hafez Bajoghli² \\ Dena Sadeghi Bahmani ${ }^{3}$ \\ Edith Holsboer-Trachsler ${ }^{3}$ \\ Serge Brand ${ }^{3,4}$ \\ 'Behavioral Disorders and Substances \\ Abuse, Research Center, Hamadan \\ University of Medical Sciences, \\ Hamadan, Iran; ${ }^{2}$ Iranian National \\ Center for Addiction Studies (INCAS), \\ Iranian Institute for Reduction of \\ High-Risk Behaviors, Tehran University \\ of Medical Sciences, Tehran, Iran; \\ ${ }^{3}$ Psychiatric Clinics of the University of \\ Basel, Center for Affective, Stress and \\ Sleep Disorders, ${ }^{4}$ Department of Sport, \\ Exercise and Health Science, Sport \\ Science Section, University of Basel, \\ Basel, Switzerland
}

Correspondence: Serge Brand Psychiatric Clinics of the University of Basel, Center for Affective, Stress and Sleep Disorders, Wilhelm Klein-Strasse 27, 4012 Basel, Switzerland

$\mathrm{Tel}+4|6| 3255114$

Fax +4I 6I 3255513

Email serge.brand@upkbs.ch
Background: For students attending university courses, experiencing test anxiety (TA) dramatically impairs cognitive performance and success at exams. Whereas TA is a specific case of social phobia, emotional intelligence (EI) is an umbrella term covering interpersonal and intrapersonal skills, along with positive stress management, adaptability, and mood. In the present study, we tested the hypothesis that higher EI and lower TA are associated. Further, sex differences were explored.

Method: During an exam week, a total of 200 university students completed questionnaires covering sociodemographic information, TA, and EI.

Results: Higher scores on EI traits were associated with lower TA scores. Relative to male participants, female participants reported higher TA scores, but not EI scores. Intrapersonal and interpersonal skills and mood predicted low TA, while sex, stress management, and adaptability were excluded from the equation.

Conclusion: The pattern of results suggests that efforts to improve intrapersonal and interpersonal skills, and mood might benefit students with high TA. Specifically, social commitment might counteract TA.

Keywords: test anxiety, emotional intelligence, students, interpersonal skills, intrapersonal skills

\section{Introduction}

University academic success is related to successfully passing exams. From a cognitive perspective, learning matter must be transferred from working memory into long-term memory from whence stored information must be retrieved and knowledge appropriately applied to specific contexts again via working memory. ${ }^{1}$ However, for some students there is a gap between their knowledge and their exam performances. ${ }^{2}$ Specifically, some students experience test anxiety (TA), a special case of social phobia. ${ }^{3}$ Students with TA often fail to pass exams or to perform at a level consistent with their knowledge because they feel unable to function cognitively and the exam seems to trigger anxiety. For example, Tektaş et $\mathrm{al}^{4}$ reported a prevalence rate of $29 \%$ of TA among German medical students, and Green et $\mathrm{al}^{2}$ reported that approximately $22 \%$ of the US medical students had moderate-to-high TA scores. Furthermore, of students who withdrew from their studies on mental grounds, $62 \%$ reported suffering from TA. ${ }^{5}$ A further observation is that compared with males, females reported higher TA scores. ${ }^{4,6-8}$

To treat TA, a broad range of cognitive-behavioral and relaxation techniques have been proposed, ${ }^{9,10}$ though Green et $\mathrm{al}^{2}$ reported only modest improvements in TA following a test taking strategy course. This finding might reflect the fact that TA is less of a cognitive or strategic issue and more of emotion regulation. In the light of this possibility, we asked about the relation between TA and so-called emotional intelligence (EI). 
EI is an umbrella term covering a range of intrapersonal and interpersonal skills as well as stress management, adaptability, and mood. ${ }^{11-13}$ We hypothesized that EI and TA would be negatively associated. Further, we predicted higher TA scores in female as compared with male participants. We also investigated which of the EI dimension would best predict TA. To this end, 200 medical students took part in the study and completed questionnaires assessing TA and EI.

\section{Method}

\section{Procedure and sample}

A total of 200 medical students (mean age $\mathrm{M}=21.05$ years; $\mathrm{SD}=1.28 ; 66 \%$ females, $\mathrm{M}_{\mathrm{f}}=21.20$ years, $\mathrm{SD}=1.34$; $\left.\mathrm{M}_{\mathrm{m}}=20.75, \mathrm{SD}=1.01 ; t(198)=2.42, P=0.02, \mathrm{~d}=0.38\right)$ of the Hamadan University of Medical Sciences (Hamadan, Iran) took part in the study. Participants were fully informed about the aims of the study. Most importantly, participants were fully informed about the voluntary basis of participation; they were also informed that questionnaires were completed anonymously, and that participation or nonparticipation would have no advantages or disadvantages for their exams and academic performance. Thereafter, participants gave their written informed consent. During the week in which the spring exams began (spring 2014), they completed a questionnaire booklet covering sociodemographic data, TA, and EI (Emotional Quotient Inventory; [see description of the instruments in the Tools section]). The Institutional Review Board of the Hamadan University of Medical Sciences approved the study, which was carried out in accordance with the ethical standards laid down in the Declaration of Helsinki.

\section{Tools}

\section{Assessing TA}

The Farsi Test Anxiety Inventory by Abolghasemi et $\mathrm{al}^{14}$ and Ejei et $\mathrm{al}^{15}$ was applied. It consists of 25 items focusing on thoughts, emotions, and behavior related to anxiety before, during, and after written and oral tests. Typical items are: "Thinking about my grade in a course interferes with my work in tests" or "I feel confident and relaxed while taking tests". Answers are given on 4-point rating scale ranging from 0 (= never) to 3 (= often), with higher sum scores reflecting increased TA (Cronbach's alpha $=0.91$ ).

\section{Assessing El}

The translated and validated Farsi version ${ }^{16}$ of the Emotional Quotient inventory ${ }^{11}$ was applied. The self-report inventory consists of 133 items. Items are declarative statements phrased in the first-person singular, and respondents are asked to indicate the degree to which the statement accurately describes them. Answers are given on a 5-point rating scale with anchor points 1 (=not true) and 5 (= true of me); higher scores reflecting higher EI. The following main factors were calculated: intrapersonal skills, interpersonal skills, stress management, adaptability, and general mood (Cronbach's alpha for the overall scale $=0.88$ ).

\section{Statistical analysis}

First, a series of $t$-tests was performed to compare TA and EI traits between female and male participants. Second, a series of correlations was performed to calculate the associations between TA and EI traits (entire sample and sex split). Third, a multiple regression analysis (stepwise, backward) was calculated to predict TA as a function of EI traits and sex. Statistics was performed with SPSS ${ }^{\circledR} 21.0$ (IBM Corporation, Armonk, NY, USA) for Apple Mac ${ }^{\circledR}$.

\section{Results}

\section{Sex differences in El and TA traits}

Table 1 reports all descriptive and inferential statistical indices to compare TA and EI scores between female and male participants. While EI scores did not differ between female and male participants, compared with male participants,

Table I Descriptive statistics and correlations between test anxiety and emotional intelligence traits

\begin{tabular}{llll}
\hline & Female patients & Male patients & Statistics \\
\hline $\mathrm{N}$ & $\mathrm{I} 32$ & 68 & \\
Emotional intelligence traits, mean (SD) & & \\
Intrapersonal skills & $23.5 \mathrm{I}(\mathrm{I} .97)$ & $23.77(2.18)$ & $t(198)=0.8 \mathrm{I}, \mathrm{d}=0.1 \mathrm{I}[\mathrm{S}]$ \\
Interpersonal skills & $23.77(1.99)$ & $23.85(2.37)$ & $t(198)=0.24, \mathrm{~d}=0.03[\mathrm{~S}]$ \\
Stress management & $23.24(2.34)$ & $23.46(2.64)$ & $t(198)=0.60, \mathrm{~d}=0.08[\mathrm{~S}]$ \\
Adaptability & $23.57(2.03)$ & $24.02(2.19)$ & $t(198)=1.43, \mathrm{~d}=0.21[\mathrm{~S}]$ \\
General mood & $23.42(2.00)$ & $23.85(2.37)$ & $t(198)=0.96, \mathrm{~d}=0.07[\mathrm{~S}]$ \\
Test anxiety & $34.37(4.49)$ & $31.60(7.42)$ & $t(198)=2.48, * \mathrm{~d}=0.56[\mathrm{M}]$ \\
\hline
\end{tabular}

Note: $* p<0.05$

Abbreviations: $M$, medium effect size; S, small effect size; SD, standard deviation. 
Table 2 Descriptive statistics and correlations between test anxiety and emotional intelligence traits, for the entire sample and separately for sex

\begin{tabular}{llll}
\hline & Sample & & \\
\cline { 2 - 4 } & Entire sample & Female participants & Male participants \\
\hline $\mathrm{N}$ & 200 & 132 & 68 \\
Emotional intelligence traits & Test anxiety & Test anxiety & Test anxiety \\
Intrapersonal skills & $r$ & $r$ & $r$ \\
Interpersonal skills & $-0.65^{* * *}$ & $-0.64^{* * *}$ & $-0.68^{* * *}$ \\
Stress management & $-0.61^{* * *}$ & $-0.54^{* * *}$ & $-0.73^{* * *}$ \\
Adaptability & $-0.55^{* * *}$ & $-0.54^{* * *}$ & $-0.57^{* * *}$ \\
General mood & $-0.56^{* * *}$ & $-0.51^{* * *}$ & $-0.61^{* * *}$ \\
\hline
\end{tabular}

Note: $* * * p<0.001$

female participants reported modest (medium effect size) but statistically higher TA scores.

\section{Associations between TA and El traits}

All descriptive and inferential statistics are reported in Table 2. Irrespective of sex, EI traits and TA were negatively associated.

\section{Predicting TA as a function of El}

To predict TA as a function of EI, a multiple regression (stepwise backward) was performed, with TA as dependent variable, and sex ( $0=$ male; $1=$ female $)$ and EI traits as predictors. The equation was as follows: $R=0.69 ; R^{2}=0.476$; Durbin-Watson coefficient $=1.52$; intrapersonal skills: $\beta=-0.23, t=3.61, P=0.001$; interpersonal skills: $\beta=-0.24$, $t=3.00, P=0.001$; general mood: $\beta=-0.20, t=2.38, P=0.05$. In other words, intra- and interpersonal skills and general mood were significant predictors of TA. Stress management ( $\beta=-0.04, t=0.23, P>0.45)$, adaptability $(\beta=-0.09, t=0.98$, $P>0.32)$, and sex $(\beta=0.12, t=1.78, P>0.21)$ were excluded from the equation.

\section{Discussion}

The key findings of the present study are that among a sample of Iranian medical students, lower TA was associated with higher EI. Specifically, better inter- and intrapersonal skills and general mood, but not stress management, adaptability, or sex predicted TA.

We hypothesized that TA and EI would be negatively associated and this was confirmed. Accordingly, our findings are consistent with the view that emotional regulation and low TA are interlinked. Further, as shown in Table 2, the pattern of results did not differ between female and male participants.

Next, we expected higher TA scores in female as compared with male participants, and this was also confirmed. Accordingly, the present pattern of results is in accordance with those results observed in schoolchildren, ${ }^{8}$ adolescents, ${ }^{6}$ and university students. ${ }^{7,17}$ However, the difference was modest (medium effect size).

Next and most importantly, results from the multiple regression analysis suggest that, statistically, "mere" emotional regulation had an influence on TA, but cognitive strategies such as stress management and adaptability did not. In this regard, we speculate that this pattern of results makes some sense of previous findings that, on the one hand, a test taking strategy course, understood as cognitive strategies for coping with TA, basically failed to reduce $\mathrm{TA},{ }^{2}$ while on the other hand, relaxation techniques such as breathing control, autogenic training, or Mindfulness-Based Stress Reduction turned out to be much more successful in reducing TA. ${ }^{9,10}$ Most importantly, it is possible to raise EI through psychotherapeutic interventions; Jahangard et al ${ }^{16}$ produced improvements in the EI of patients with borderline personality disorders after teaching EI, relative to a control condition.

The quality of the data does not allow a deeper understanding of the underlying psychological mechanisms, although we speculate that theories by Eysenck et $\mathrm{al}^{18} \mathrm{might}$ be able to explain, why cognitive performance dramatically decreases with TA: Eysenck et al ${ }^{18}$, Eysenck and Byrne ${ }^{19}$, Eysenck and Calvo ${ }^{20}$ proposed a "mere" cognitive model in which cognitive resources and attention needed to solve exam questions allocated to cope with anxiety and current concerns. ${ }^{1}$ While there is basically no contradiction between the model by Eysenck et al ${ }^{18}$ and the present results, we speculate and believe that reducing TA requires not only techniques to improve working memory, but also require techniques to reduce anxiety. In this respect, speculatively, it also seems likely that improving students' intra- and interpersonal skills would be more successful in reducing TA. ${ }^{16}$ 
In our view, this is also consistent with research on stress and hardiness, ${ }^{21}$ in which commitment, understood as the feeling of being related to and responsible for those close to the self, has been found to be one of the main factors for both preventing and coping with stress.

Despite the intriguing findings, the following caveats warrant against overgeneralization. First, the sample was rather small, and medical students might be under greater pressure to achieve in exams because of the rigorous selection involved. Second, only students able and willing participated in the study. Third, we relied entirely on self-reports, whereas experts' ratings might have allowed further insight into possible psychiatric disorders and comorbidities. Fourth, the present pattern of results might have emerged due to further latent, but unassessed variables, which might have biased two or more dimensions in the same direction. Fifth, participants completed anonymously the questionnaire before starting the exams. As a result, the present data of EI and TA could not be matched with the results of the exams (pass/fail; grades). Future studies should compare dimensions of EI and TA with the objective performance.

\section{Conclusion}

Among a sample of Iranian students, irrespective of sex, lower TA and higher EI were associated. The pattern of results suggests that improving students' interpersonal and intrapersonal skills might reduce TA.

\section{Acknowledgment}

The authors would like to thank Nick Emler (University of Surrey, Surrey, UK) for proofreading the manuscript.

\section{Disclosure}

The authors report no conflicts of interest in this work.

\section{References}

1. Sternberg RJ, Sternberg K. Cognitive Psychology. 6th ed. Belmont, CA: Wadsworth; 2009.

2. Green M, Angoff N, Encandela J. Test anxiety and United States Medical Licensing Examination scores. Clin Teach. Epub 2015 Jun 3.
3. American Psychiatric Association. Diagnostic and Statistical Manual of Mental Disorders (DSM 5). 5th ed. Washington, DC: American Psychiatric Association; 2013.

4. Tektaş OY, Paulsen F, Sel S. Test anxiety among German medical students and its impact on lifestyle and substance abuse. Med Teach. 2013;35(11):969.

5. National Alliance Mental Illness (NAMI). College students speak. A survey report on mental health. Available from: https://www.nami. org/About-NAMI/Publications-Reports/Survey-Reports/CollegeStudents-Speak_A-Survey-Report-on-Mental-H.pdf. Accessed August 10, 2015.

6. Harpell JV, Andrews JJW. Relationship between school based stress and test anxiety. Int J Psychol Stud. 2013;5(2):74-84.

7. Mohammadyari G. Comparative study of relationship between general perceived self-efficacy and test anxiety with academic achievement of male and female students. Procedia Soc Behav Sci. 2012;69: 2119-2123.

8. Putwain DW. Test anxiety in UK schoolchildren: prevalence and demographic patterns. Br J Educ Psychol. 2007;77(3):579-593.

9. Vøllestad J, Nielsen MB, Nielsen GH. Mindfulness- and acceptancebased interventions for anxiety disorders: a systematic review and meta-analysis. Br J Clin Psychol. 2012;51(3):239-260.

10. Li AW, Goldsmith CA. The effects of yoga on anxiety and stress. Altern Med Rev. 2012;17(1):21-35.

11. Bar-On R. Emotional Quotient Inventory: Technical Manual. Toronto, ON: Multi-Health Systems; 1997.

12. Bar-On R. The Bar-On model of emotional-social intelligence (ESI). Psicothema. 2006;18(1):13-25.

13. Bar-On R. The Bar-On Emotional Quotient Inventory (EQ-i): rationale, description and psychometric properties. In: Geher G, editor. Measuring Emotional Intelligence: Common Ground and Controversy. Hauppauge, NY: Nova Science; 2004.

14. Abolghasemi A, Asadi Moghadam A, Nadjarian B, Shokrkon H. Build and validate a scale to measure anxiety in the third grade students in middle school of Ahwaz. J Educ Psychol. 1996;3-4:61-74.

15. Ejei J, Rezaei MR, Lavasani MG. The effectiveness of coping strategies training with irrational beliefs (cognitive approach) on test anxiety in students. Procedia Soc Behav Sci. 2011;30:2165-2168.

16. Jahangard L, Haghighi M, Bajoghli H, et al. Training emotional intelligence improves both emotional intelligence and depressive symptoms in inpatients with borderline personality disorder and depression. Int J Psychiatry Clin Pract. 2012;16(3):197-204.

17. Mousavi M, Haghshenas H, Alishahi MJ. Effect of gender, school performance and school type on test anxiety among Iranian adolescents. Iran Red Crescent Med J. 2008;10(1):4-7.

18. Eysenck MW, Derakshan N, Santos R, Calvo MG. Anxiety and cognitive performance: attentional control theory. Emotion. 2007;7(2): 336-353.

19. Eysenck MW, Byrne A. Anxiety and susceptibility to distraction. Pers Ind Diff. 1992;13(7):793-798.

20. Eysenck MW, Calvo MG. Anxiety and performance: the processing efficiency theory. Cogn Emot. 1992;6(6):409-434.

21. Maddi SR, Kobasa SC. The Hardy Executive: Health Under Stress. Homewood, IL: Dow Jones-Irwin; 1984.
Neuropsychiatric Disease and Treatment

\section{Publish your work in this journal}

Neuropsychiatric Disease and Treatment is an international, peerreviewed journal of clinical therapeutics and pharmacology focusing on concise rapid reporting of clinical or pre-clinical studies on a range of neuropsychiatric and neurological disorders. This journal is indexed on PubMed Central, the 'PsycINFO' database and CAS,

\section{Dovepress}

and is the official journal of The International Neuropsychiatric Association (INA). The manuscript management system is completely online and includes a very quick and fair peer-review system, which is all easy to use. Visit http://www.dovepress.com/testimonials.php to read real quotes from published authors. 